\title{
Antibacterial activity and biological performance of a novel antibacterial coating containing a halogenated furanone compound loaded poly(L-lactic acid) nanoparticles on microarc-oxidized titanium
}

\author{
This article was published in the following Dove Press journal: \\ International Journal of Nanomedicine \\ 19 January 2015 \\ Number of times this article has been viewed
}

\author{
Yicheng Cheng',2,* \\ Xianghui Zhao ${ }^{3, *}$ \\ Xianghui Liu ${ }^{2, *}$ \\ Weige Sun ${ }^{2}$ \\ Huifang Ren' \\ Bo Gao' \\ Jiang $\mathrm{Wu}^{\prime}$
}

'State Key Laboratory of Military Stomatology, Department of Prosthodontics, School of Stomatology, Fourth Military Medical University, Xi'an, People's Republic of China;

${ }^{2}$ Department of Stomatology, Jingdu Hospital, Nanjing, People's Republic of China; ${ }^{3}$ nstitute of Neuroscience, School of Basic Medicine, Fourth Military Medical University, Xi'an, People's Republic of China

*These authors contributed equally to this work

Correspondence: Bo Gao State Key Laboratory of Military Stomatology, Department of Prosthodontics, School of Stomatology, Fourth Military Medical University, No 145, West Changle Road, Xi'an, Shaanxi 710032, People's Republic of China

Tel +86298477 6469

Fax +862984776469

Email gaobo@fmmu.edu.cn

Jiang Wu

State Key Laboratory of Military

Stomatology, Department of

Prosthodontics, School of Stomatology,

Fourth Military Medical University, No

145, West Changle Road, Xi'an, Shaanxi

710032, People's Republic of China

Tel +86298477 6469

Fax +86298477 6469

Email wujiang@fmmu.edu.cn

\begin{abstract}
Titanium implants have been widely used for many medical applications, but bacterial infection after implant surgery remains one of the most common and intractable complications. To this end, long-term antibacterial ability of the implant surface is highly desirable to prevent implant-associated infection. In this study, a novel antibacterial coating containing a new antibacterial agent, (Z-)-4-bromo-5-(bromomethylene)-2(5H)-furanone loaded poly(L-lactic acid) nanoparticles, was fabricated on microarc-oxidized titanium for this purpose. The antibacterial coating produced a unique inhibition zone against Staphylococcus aureus throughout a 60-day study period, which is normally long enough to prevent the infection around implants in the early and intermediate stages. The antibacterial rate for adherent $S$. aureus was about $100 \%$ in the first 10 days and constantly remained over $90 \%$ in the following 20 days. Fluorescence staining of adherent $S$. aureus also confirmed the excellent antibacterial ability of the antibacterial coating. Moreover, in vitro experiments showed an enhanced osteoblast adhesion and proliferation on the antibacterial coating, and more notable cell spread was observed at the early stage. It is therefore concluded that the fabricated antibacterial coating, which exhibits relatively long-term antibacterial ability and excellent biological performance, is a potential and promising strategy to prevent implant-associated infection.
\end{abstract}

Keywords: microarc oxidation, osteoblasts

\section{Introduction}

Titanium (Ti) implants continue to play essential roles in the repair or replacement of bony tissues that have become diseased or damaged. ${ }^{1}$ However, one of the most common and intractable complications during this process is the implant-associated infection, ${ }^{2}$ which may lead to various serious consequences, such as prolonged hospitalization, implant failure, reoperation, osteomyelitis, amputation, and even death. ${ }^{3}$ Although the risk of such infection can be minimized by thorough disinfection and rigorous aseptic operation, the percentage of postoperation infections is still up to $3 \%{ }^{4}$ Once infections occur, bacteria tend to form a biofilm on the implant surface, which protects them from being completely eliminated by antibacterial agents coming from outside. ${ }^{5}$ Therefore, fabricating antibacterial coatings on the surface of Ti implants is an effective approach to prevent initial bacterial adhesion around implants, in the pathogenesis of infection. ${ }^{6}$ However, most attempts at this have failed to sustain long-term antibacterial effects. ${ }^{7}$ Further, the emerging appearance of insusceptibility of bacteria and drug resistance also encourages the search for novel and more effective antibacterial strategies. ${ }^{8}$ 
Halogenated furanones, originally extracted from the red alga Delisea pulchra, have been proven to possess potent antimicrobial effects against a great number of bacterial species and to inhibit biofilm formation. ${ }^{9}$ They are structurally similar to bacterial N-acyl homoserine lactones (AHLs), which participate in cell-to-cell communication to control swarming and biofilm formation in various microbial species. ${ }^{10}$ Thus, halogenated furanones acting as AHL antagonists have negative impacts on bacterial colonization. This antimicrobial mechanism would render halogenated furanones less prone to the development of drug resistance. ${ }^{11}$ Many studies have been designed to evaluate the effects of halogenated furanones on eukaryotic cell viability, and there was few data reported the toxicity of halogenated furanones. ${ }^{12,13}$ On the aforementioned basis, halogenated furanone compounds have been considered as potential and ideal antibacterial agents for medical device coating., ${ }^{9,12}$

For the past decade, special attention has been paid to polymeric microsphere/nanoparticles (NPs) for their sustained-release effects. ${ }^{14,15}$ Poly(D, L-lactic acid) (PLA) has been widely developed as drug carrier, due to its desirable biocompatibility and biodegradability. ${ }^{16}$ Particularly, it has been approved by US Food and Drug Administration for clinic use.

Microarc oxidation (MAO) treatment is a relatively convenient and effective technique for preparing $\mathrm{TiO}_{2}$-based coatings on Ti substrate. MAO coating exhibits a porous structure, which has been proved to be beneficial to osteoblast adhesion and proliferation. ${ }^{17,18}$ Currently, MAO is being widely applied for creating bioactive composite coatings on $\mathrm{Ti}^{19,20}$

By optimizing the preparation parameters, a halogenated furanone compound, (Z-)-4-bromo-5-(bromomethylene)2(5H)-furanone (BBF)-loaded poly(L-lactic acid) (PLLA) NPs (BBF/PLLA-NPs) with high encapsulation efficiency and long-term release behavior were prepared, using the oil-in-water emulsion solvent evaporation method in our previous study. ${ }^{21}$ After this, we developed a method to fabricate a new antibacterial coating, by cross-linking the BBF/PLLA-NPs on microarc-oxidized titanium (MAO-Ti), and the new antibacterial coating sustained its release of $\mathrm{BBF}$ for about 60 days. ${ }^{22}$ In this scenario, the current study was specifically designed to further investigate the actual antibacterial capability and the biological performance of the fabricated antibacterial coating.

\section{Materials and methods}

Fabrication of the antibacterial coating and study of its release effects

Preparation of BBF-PLLA-NPs

BBF-PLLA-NPs, composed ofBBF ( $\geq 97.0 \%$ )(Sigma-Aldrich Corp, St Louis, MO, USA) and PLLA (average molecular weight $[\mathrm{MW}]=152,000$ ) (Sigma-Aldrich Corp), were prepared by the oil-in-water emulsion solvent evaporation method as described elsewhere. ${ }^{21}$ In brief, $15 \mathrm{mg}$ BBF along with $100 \mathrm{mg}$ PLLA were dissolved in $2 \mathrm{~mL}$ dichloromethane (DCM) to form the oil phase. This oil phase was added dropwise into $40 \mathrm{~mL}$ of $1 \%$ (w/v) poly (vinyl alcohol) (PVA) (Sigma-Aldrich Corp) aqueous solution. The mixture solution was then probe-sonicated using a Sonifier Cell Disrupter (JY98-IIIN; Ningbo Xingzhi Biological Technology Co., Ltd, People's Republic of China) at the intensity of $300 \mathrm{~W}$ for 200 seconds, in an ice bath, to produce an oil-in-water emulsion. DCM was evaporated completely by magnetic stirring for 6 hours at $25^{\circ} \mathrm{C}$ under reduced pressure. The produced NPs were collected by ultracentrifuge (12,000 rpm, 20 minutes, $4^{\circ} \mathrm{C}$ ), followed by rinsing with distilled water. The shape and surface morphology of the BBF-PLLA-NPs were observed by scanning electron microscope (SEM) (S-4800; Hitachi Co., Tokyo, Japan). The PLLA-NPs were prepared by the same method, except that the oil phase did not contain BBF.

\section{MAO treatment}

Square-shaped $(10 \times 10 \times 2 \mathrm{~mm})$ and disk-shaped $(6 \mathrm{~mm}$ in diameter and $2 \mathrm{~mm}$ in thickness) commercially pure Ti (cp-T)I (>99.5 wt\%, grade 2) specimens were polished, ultrasonically cleaned, and air-dried. MAO treatment was carried out using a pulsed direct current power supply (at Xi'an University of Technology, Xi'an, People's Republic of China) in a mixed aqueous solution containing $0.04 \mathrm{M} \beta$-glycerophosphate sodium and $0.2 \mathrm{M}$ calcium acetate. The applied voltage, frequency, duty cycle, and oxidizing time were $300 \mathrm{~V}, 600 \mathrm{~Hz}$, $8.0 \%$, and 5 minutes, respectively. The surface morphology of MAO-Ti was observed by SEM, and the element composition was analyzed by associated energy dispersive spectroscope (EDS) (Oxford Instruments, Abingdon, UK).

\section{Fabrication of the antibacterial coating and study of its release effects}

The antibacterial coating was fabricated by cross-linking BBF-PLLA-NPs with gelatin on MAO-Ti. ${ }^{22}$ Briefly, $20 \mathrm{mg}$ BBF-PLLA-NPs were sonicated for 20 minutes and dispersed in $5 \mathrm{~mL} 0.2 \%$ gelatin solution $(\mathrm{w} / \mathrm{v})$, and $400 \mu \mathrm{L}$ of the suspension was dropped onto the square MAO-Ti specimen, while $113 \mu \mathrm{L}$ of the suspension was dropped onto the disk specimen. The specimens were then oscillated on an oscillator for 1 hour to enable the NPs to penetrate the pores on the MAO-Ti. Subsequently, these were dried at $4^{\circ} \mathrm{C}$ and then immersed in $2.5 \%(\mathrm{w} / \mathrm{v})$ glutaraldehyde solution for 30 minutes to cross-link the gelatin. Finally, the specimens were washed with ethanol to remove the remaining 
glutaraldehyde and freeze-dried. The surface morphology of the antibacterial coating was observed by SEM.

BBF release from the antibacterial coating was achieved by placing one specimen with $5 \mathrm{~mL}$ phosphate-buffered saline (PBS) in a dialysis bag (MW cutoff at $3,500 \mathrm{~g} / \mathrm{mol}$ ). This dialysis bag was then immersed into $20 \mathrm{~mL}$ PBS, with continuous gently magnetic stirring $(60 \mathrm{rpm})$ at $37^{\circ} \mathrm{C}$. Next, $1 \mathrm{~mL}$ aliquots were withdrawn and replaced with the same volume of fresh PBS at predetermined time intervals. The BBF concentration in PBS was measured using ultraviolet spectroscopy (UV2550; Shimadzu, Kyoto, Japan) at $286.0 \mathrm{~nm}$, which is the characteristic excitation wavelength for BBF. The measurements were repeated in triplicate.

\section{Characterization of the test groups}

Based on the different tests, the specimens were divided into four groups: (1) BBF/PLLA-MAO-Ti, with BBF-PLLA-NPs cross-linked with gelatin on MAO-Ti; (2) PLLA-MAO-Ti, with PLLA-NPs cross-linked with gelatin on MAO-Ti;

(3) MAO-Ti; and (4) polished cp-Ti.

Four roughness parameters were selected to depict the surface roughness: average roughness $(\mathrm{Ra})$, range of the roughness (Rt), peak-to-valley roughness $(\mathrm{Rz})$, and peakto-mean-height roughness (Rp), by surface profilometer (TR240; TIME Group, People's Republic of China). Surface hydrophilicity was evaluated by measuring the water contact angle, using a sessile drop method on the Drop Shape Analysis System DSA 10 (Kruss, Hamburg, Germany) and using deionized water at room temperature. The measurements were repeated at nine different sites for each specimen.

\section{Antibacterial assay Inhibition zones}

The inhibition zones of the specimens were determined according to disk diffusion assay. ${ }^{23}$ Staphylococcus aureus (ATCC 25923; ATTC, Manassas, VA, USA), cultivated on Mueller-Hinton agar (MHA) plates at $37^{\circ} \mathrm{C}$ for 24 hours, was used in this assay. It was adjusted to match a tube of 0.5 McFarland turbidity standard, using the spectrophotometer at $600 \mathrm{~nm}$, which was equal to $1.5 \times 10^{8} \mathrm{CFU} / \mathrm{mL}$. The surface of the MHA plates was completely inoculated using a sterile cotton swab steeped in the prepared suspension of bacterium. The four disk specimens were then aseptically placed over the inoculated plates. Finally, the plates were incubated at $37^{\circ} \mathrm{C}$ for 1 day, and the diameter of the inhibition zones was measured. The MHA plates were replaced by new inoculated plates every day, and the specimens were ultrasonically cleaned, dried, sterilized, and repeatedly reincubated on the replaced MHA for a total of 65 days.

\section{Antibacterial rate (AR)}

The $S$. aureus was adjusted to a concentration of $10^{6} \mathrm{CFU} / \mathrm{mL}$ in Mueller-Hinton broth. Each square specimen was incubated in $1 \mathrm{~mL}$ of the bacteria suspension, at $37^{\circ} \mathrm{C}$, for 1 day. After incubation, the bacteria attached on the various specimens were gently rinsed with PBS and ultrasonically detached, using $10 \mathrm{~mL}$ of PBS solution, for 5 minutes. The bacterial suspensions were recultivated on MHA plates for colony counting. The AR was calculated by the following formula:

\section{$\mathrm{AR}(\%)=(\mathrm{CFU}$ of control $-\mathrm{CFU}$ of experimental groups $) /$} CFU of control $\times 100 \%$,

where polished cp-Ti served as the control, and BBF/PLLAMAO-Ti, PLLA-MAO-Ti, and MAO-Ti constituted the experimental groups. The specimens were also ultrasonically cleaned, dried, sterilized, and reincubated for 65 days as described above.

\section{Viability of bacteria}

After exposure to bacteria for 1 day as aforementioned in the section "Antibacterial rate", the specimens were rinsed thrice with PBS and stained with SYTO $^{\circledR} 9$ and propidium iodide dyes (Molecular Probes LIVE/DEAD ${ }^{\circledR}$ BacLight $^{\mathrm{TM}}$ Bacterial Viability Kits; Life Technologies Corp, Carlsbad, CA, USA) for 15 minutes in the dark, followed by examination using laser scanning confocal microscopy (FV1000; Olympus, Tokyo, Japan). The green-fluorescent SYTO 9 and red-fluorescent propidium iodide differ in their abilities to penetrate bacteria. When both dyes are employed, the live bacteria (with intact membranes) were stained by SYTO 9 (green), and the dead bacteria (with damaged membranes) were stained by propidium iodide (red).

\section{In vitro test of the biological performance Cell culture}

Osteoblasts were isolated via sequential trypsin-collagenase digestion of neonatal rat calvaria according to the established protocol. ${ }^{24}$ The cells were cultured in Dulbecco's Modified Eagle's Medium (DMEM) (Gibco ${ }^{\circledR}$; Life Technologies Corp) containing 10\% fetal bovine serum (Gibco; Life Technologies Corp) in a humidified atmosphere of $5 \% \mathrm{CO}_{2}$ at $37^{\circ} \mathrm{C}$. The BBF/PLLA-MAO-Ti, MAO-Ti, and polished cp-Ti specimens were measured in these tests.

\section{Cell attachment and proliferation}

Osteoblasts were seeded at a density of $2 \times 10^{4}$ cells $/ \mathrm{cm}^{2}$ in a 24-well plate onto each square specimen. A 3-(4, 
5-Dimethylthiazol-2-yl)-2,5-diphenyltetrazolium bromide (MTT) assay was used to evaluate cell attachment at 30, 60, and 120 minutes, and cell proliferation at 1, 3, 5, and 7 days. The MTT solution $(5 \mathrm{mg} / \mathrm{mL}$ and $100 \mathrm{~mL}$ ) (Sigma-Aldrich Corp) was added to each well and incubated at $37^{\circ} \mathrm{C}$ for 4 hours. The dark-blue crystals were then dissolved by adding $1,000 \mu \mathrm{L}$ dimethyl sulfoxide (DMSO) (Hongsheng, People's Republic of China), and triplicate $200 \mu \mathrm{L}$ aliquots were transferred to a 96-well plate. The optical density was determined spectrophotometrically at $570 \mathrm{~nm}$. The blank reference was taken from an ethanol-DMSO only solution.

\section{Cell morphology}

After being cultured for 1 and 3 days, the specimens were rinsed thrice with $\mathrm{PBS}$, fixed with $3 \%$ glutaraldehyde at $4{ }^{\circ} \mathrm{C}$ for 2 hours, and dehydrated in a series of ethanol solutions for 10 minutes each. Then, the specimens were dried, platinumcoated, and examined by SEM to observe the morphological spread of the osteoblasts.

\section{Statistical analysis}

Each assay in the whole study was repeated three times, and data were expressed as mean \pm standard deviation. A one-way analysis of variance (ANOVA) combined with a StudentNewman-Keuls (SNK) post hoc test was used to determine the level of significance. Differences were considered statistically significant at $P<0.05$.

\section{Results}

\section{Fabrication of antibacterial coating and its release effects}

The BBF-PLLA-NPs were spherical in shape without any aggregation, and the particle size distribution ranged from 300 to $700 \mathrm{~nm}$, as shown in Figure 1A. At high magnification, the BBF-PLLA-NPs exhibited a smooth surface, on which no pores or cracks were observed (Figure 1B). After MAO treatment, a porous morphology, with pores 1-3 $\mu \mathrm{m}$ in the diameter, appeared on the surface of the MAO-Ti, in which $\mathrm{Ca}$ and $\mathrm{P}$ were detected; the $\mathrm{Ca} / \mathrm{P}$ ratio was about 1.54 (Figure 1C).

Figure 1D and E show the surface morphology of the antibacterial coating. The pores on MAO-Ti were almost filled by the cross-linked nanospheres, whereas only sporadic nanospheres were attached to the flat areas among the pores (Figure 1D). The BBF-PLLA-NPs were well-distributed in the pores, and were tightly cross-linked with each other and with the wall of pores, by gelatin (Figure 1E). The in vitro release profile of BBF from the antibacterial coating exhibited a biphasic release phenomenon (Figure 1F). There was a burst release of small amounts of BBF (9.4\%) into PBS in the first 4 hours. This rapid liberation was followed by a slow and sustained further release. In this sustained-release period, $80.8 \%$ of BBF was released during the following 60 days.

\section{Surface roughness and surface hydrophilicity}

Roughness parameters in the four experimental groups were compared and demonstrated in Figure 2A. The roughness of MAO-Ti was much higher than that of polished cp-Ti, and the former declined slightly after the nanospheres had filled in the pores. From the contact angle results obtained with deionized water (Figure 2B), the MAO surface was more hydrophilic than was the polished surface, and this was further increased after the nanospheres cross-linked with gelatin in the pores. No statistical differences were observed on the roughness and hydrophilicity between BBF/PLLA-MAO-Ti and PLLA-MAO-Ti $(P>0.05)$.

\section{Antibacterial activity}

The representative images of the inhibition zones from day 1 to day 65 are presented in Figure 3, which shows that BBF/ PLLA-MAO-Ti produced a unique inhibition zone against $S$. aureus throughout a 60-day period, whereas the other three specimens did not exert such an effect. The boundary of the inhibition zone at day 60 was slightly obscure (Figure 3E). The diameter changes of the inhibition zones of BBF/PLLA-MAO-Ti are recorded in Figure 4. It was apparent that the inhibition zone was biggest at the first day, and there were no obvious changes observed from day 2 to day 45 . Subsequently, the diameter of inhibition zones decreased significantly from day 45 to day 60 .

The AR for adherent bacteria on the specimens for as long as 65 days is shown in Figure 5. The MAO-Ti had an AR value of about $-270 \%$, which was constant with time. The AR value of PLLA-MAO-Ti was about - $195 \%$ during the first 45 days and then decreased. When compared with PLLA-MAO-Ti and MAO-Ti, the BBF/PLLA-MAO-Ti specimens possessed a positive and high AR value of about $100 \%$ during the first 10 days and then decreased slightly. In the next 20 days, it remained over $90 \%$. After 45 days, the AR value of BBF/PLLA-MAO-Ti dropped to $80.9 \%$ and began to decrease significantly. The BBF/PLLA-MAO-Ti showed an AR value of $30.1 \%$ at day 60 , and the AR value was negative $(-185 \%)$ at day 65 .

The viability of bacteria on the four specimens was also determined by fluorescence staining as shown in Figure 6. 

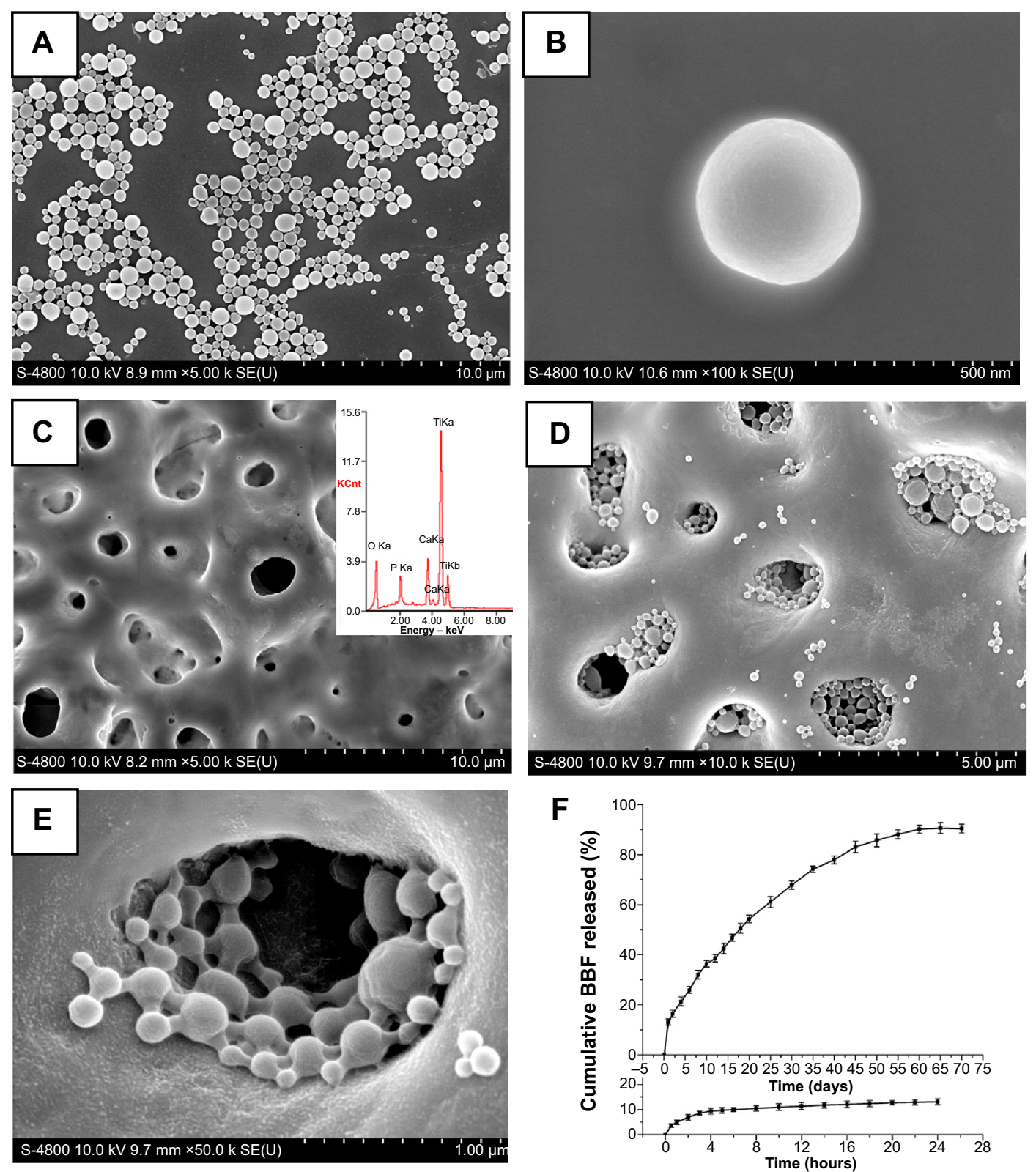

Figure I (A) Scanning electron microscope image of BBF-PLLA-NPs at low magnification. (B) Scanning electron microscope image of BBF-PLLA-NPs at high magnification. (C) Scanning electron microscope image and energy dispersive spectroscope spectrum of MAO-Ti specimen. (D) Scanning electron microscope image of the antibacterial coating at low magnification. (E) Scanning electron microscope image of the antibacterial coating at high magnification. (F) In vitro release curve of BBF from the antibacterial coating in PBS.

Abbreviations: BBF, (Z-)-4-bromo-5-(bromomethylene)-2(5H)-furanone; BBF-PLLA-NPs, (Z-)-4-bromo-5-(bromomethylene)-2(5H)-furanone loaded poly(L-lactic acid) nanoparticles; MAO-Ti, microarc-oxidized titanium; PBS, phosphate-buffered saline.

After bacterial invasion, there were large amounts of viable bacteria (green fluorescence) on the polished cp-Ti, MAO-Ti, and PLLA-MAO-Ti (Figure 6A-C). In comparison, only small amounts of dead bacteria (red fluorescence) could be observed on BBF/PLLA-MAO-Ti (Figure 6D).

\section{Biological performance}

The number of viable cells attached onto polished cp-Ti, MAO-Ti, and BBF/PLLA-MAO-Ti specimens increased from 30 minutes to 120 minutes (Figure 7A). Subsequent statistical analysis showed that the number of viable cells attached onto MAO-Ti and BBF/PLLA-MAO-Ti was higher than that on the polished surface $(P<0.05)$. Moreover, $\mathrm{BBF} /$ PLLA-MAO-Ti exhibited slightly better cell adhesion than did MAO-Ti, although not statistically significant $(P>0.05)$. Similar to cell adhesion, cell proliferation of the specimens also increased from 1 day to day 7 (Figure 7B). MAO-Ti and BBF/PLLA-MAO-Ti showed better cell proliferation 
A

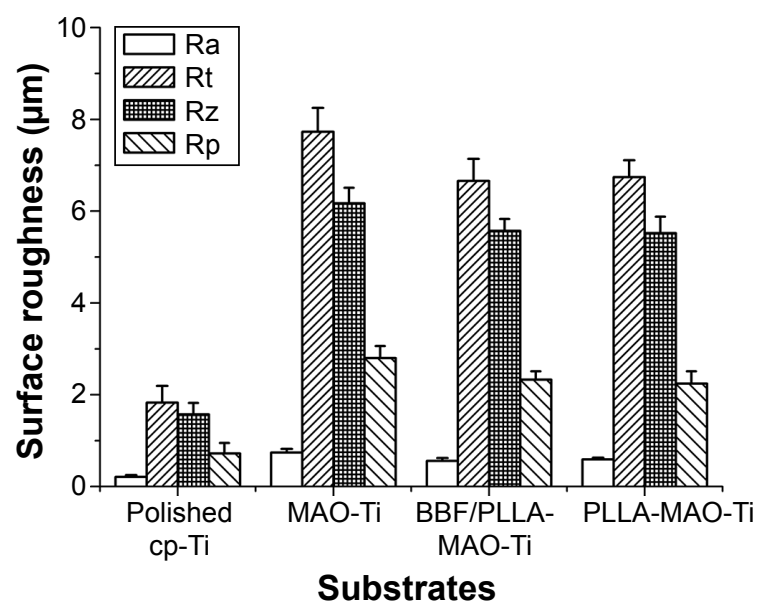

B

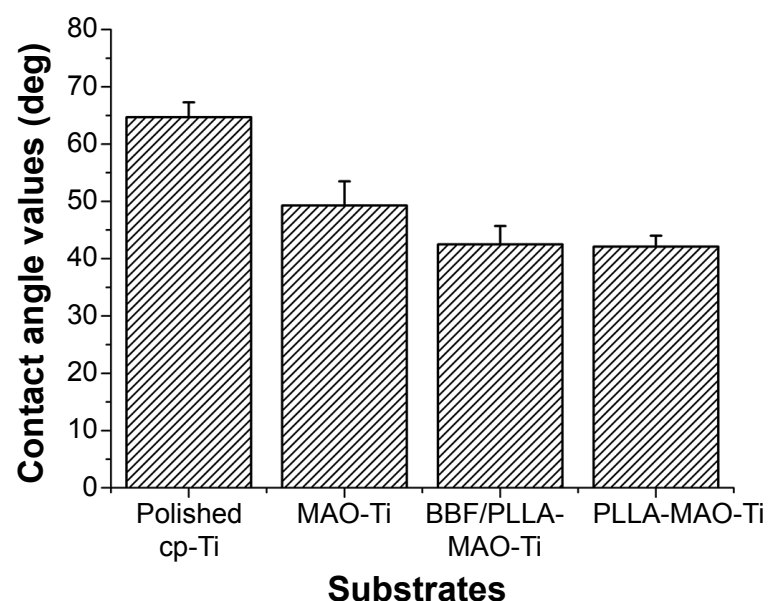

Figure 2 (A) Surface roughness parameters of the specimens. (B) Contact angle of the specimens.

Abbreviations: BBF/PLLA-MAO-Ti, cross-linking (Z-)-4-bromo-5-(bromomethylene)-2(5H)-furanone loaded poly(L-lactic acid) nanoparticles with gelatin on microarcoxidized titanium; cP-Ti, commercially pure Ti; deg, degrees; MAO-Ti, microarc-oxidized titanium; PLLA-MAO-Ti, cross-linking poly(L-lactic acid) nanoparticles with gelatin on microarc-oxidized titanium; Ra, average roughness; Rp, peak-to-mean-height roughness; Rt, range of the roughness; Rz, peak-to-valley roughness.

when compared with polished cp-Ti $(P<0.05)$. There was no statistical difference in the cell proliferation between MAO-Ti and BBF/PLLA-MAO-Ti $(P>0.05)$.

Figure 8 shows the morphology of rat osteoblasts spread on the three kinds of specimens, after 1 and 3 days. Generally, the osteoblasts were well spread on all the three surfaces. After 1 day of culture, cells grown on the polished cp-Ti and MAO-Ti showed a fusiform morphology, with some filopodia and lamellipodia extensions (Figure 8A and B). The number of lamellipodia increased slightly on BBF/PLLA-MAO-Ti
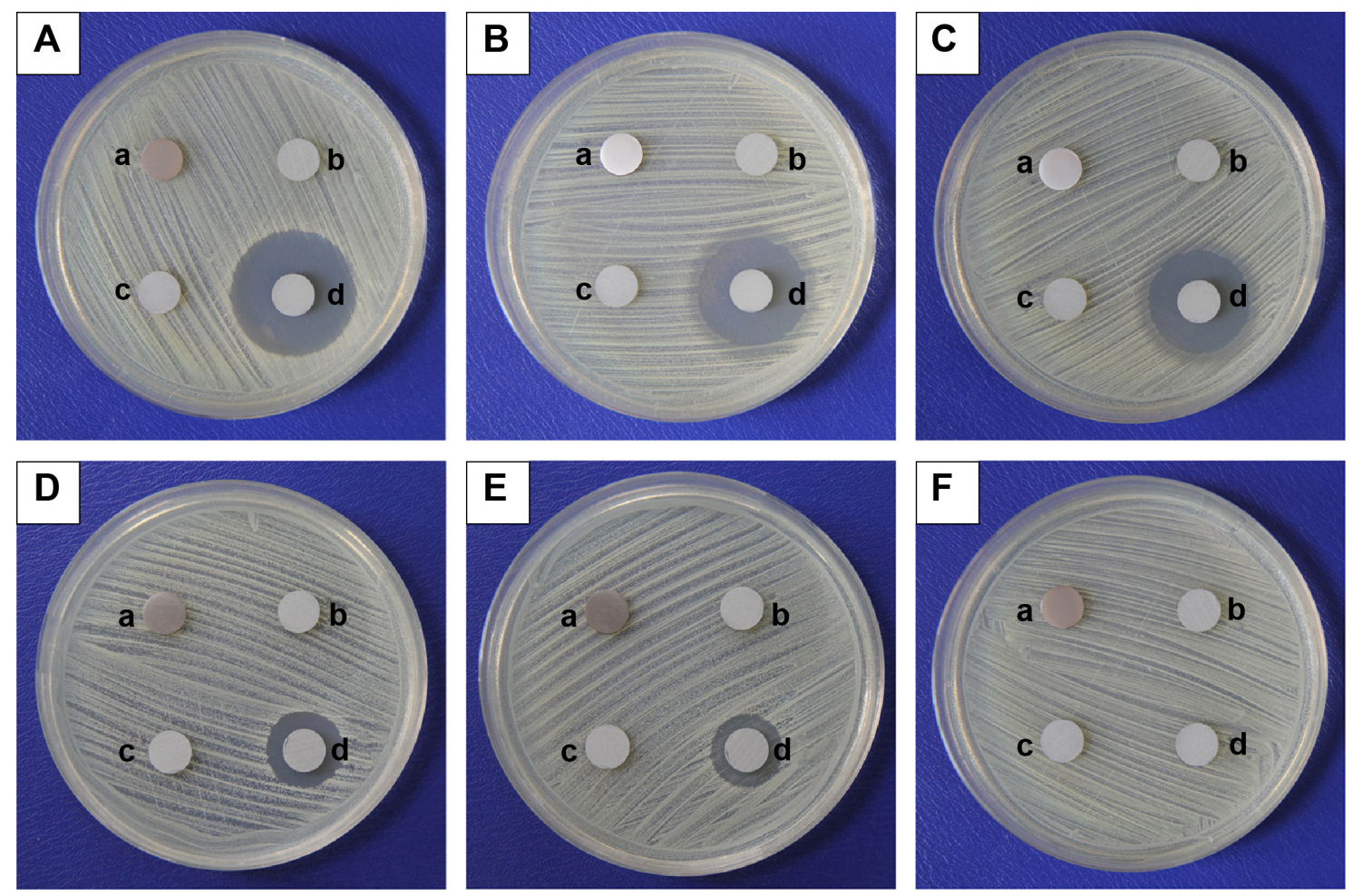

Figure 3 Representative images of inhibition zones against Staphylococcus aureus, at (A) day I, (B) day 2, (C) day 45, (D) day 55, (E) day 60, and (F) day 65. Notes: The substrates were: (a) polished cp-Ti; (b) MAO-Ti; (c) PLLA-MAO-Ti; and (d) BBF/PLLA-MAO-Ti.

Abbreviations: BBF/PLLA-MAO-Ti, cross-linking (Z-)-4-bromo-5-(bromomethylene)-2(5H)-furanone loaded poly(L-lactic acid) nanoparticles with gelatin on microarcoxidized titanium; cp-Ti, commercially pure Ti; MAO-Ti, microarc-oxidized titanium; PLLA-MAO-Ti, cross-linking poly(L-lactic acid) nanoparticles with gelatin on microarcoxidized titanium. 


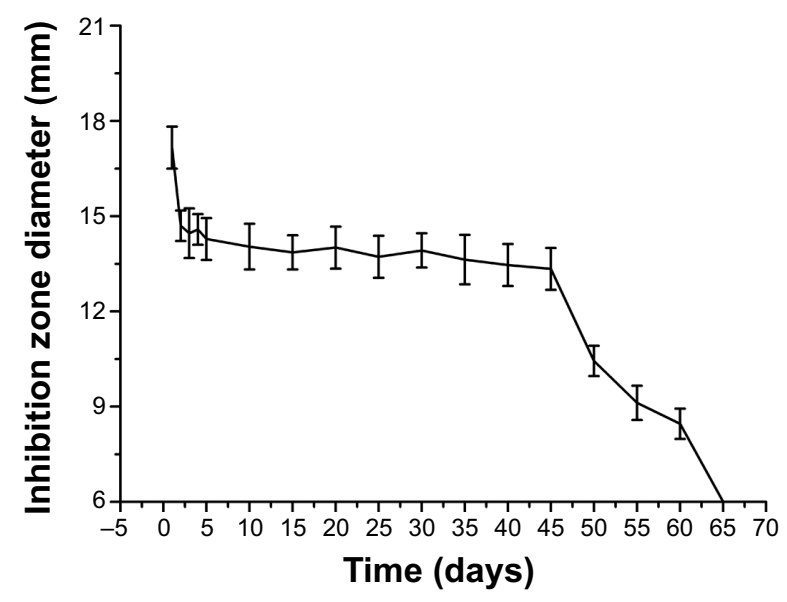

Figure 4 Diameter changes of the inhibition zones of the antibacterial coating, over 65 days.

after 1 day of culture (Figure 8C). At day 3 of culture, lamellar cells with some filopodia and lamellipodia extensions were observed on the surface of the polished cp-Ti (Figure 8D). The number of cell filopodia and lamellipodia increased significantly after 3-day culture on MAO-Ti (Figure 8E), while well-flattened cells with numerous lamellipodia were observed on BBF/PLLA-MAO-Ti (Figure 8F).

\section{Discussion}

Although Ti implants have been widely and successfully used in both orthopedic and dental clinics, infection prevention remains a continuous challenge for their clinical applications. ${ }^{25}$ Considering the susceptibility of the implant surface to bacterial adhesion, which may cause implantassociated infection, antibacterial coatings have been traditionally designed to prevent the initial bacterial adhesion. ${ }^{26}$ Long-term antibacterial ability is especially significant for implants because of the constant risk of bacterial infection. Halogenated furanones can effectively possess stable antimicrobial activity against a great number of bacteria, meanwhile they are less prone to resistance development. BBF, used in this study, is one of the most active halogenated furanone compounds. ${ }^{27}$ To achieve the goal of sustained release, we incorporated BBF into PLLA-NPs. Under the SEM, the BBF-PLLA-NPs showed a smooth surface without any pores or cracks (Figure 1B). Surface morphology is crucial for microspheres/NPs since in vitro experiments have shown that the initial burst release from a smooth surface is less than that from a rough surface. ${ }^{28,29}$ Moreover, the particle size (300-700 nm) (Figure 1A) was much smaller than the diameter of pores on MAO-Ti (1-3 $\mu \mathrm{m})$ (Figure 1C), which made it possible for the nanospheres to penetrate the pores. In addition to the physical inlay, the cross-linking gelatin could effectively prevent the antibacterial nanospheres from falling off during the insertion of implants. Therefore, this method provides a promising strategy with respect to the fabrication of long-term antibacterial coating on Ti implants.

The antibacterial coating produced a unique inhibition zone against $S$. aureus, suggesting that only the antibacterial coating possessed the antibacterial ability. The diameter changes of the inhibition zones (Figure 4) were almost consistent with the release curve of BBF from the antibacterial

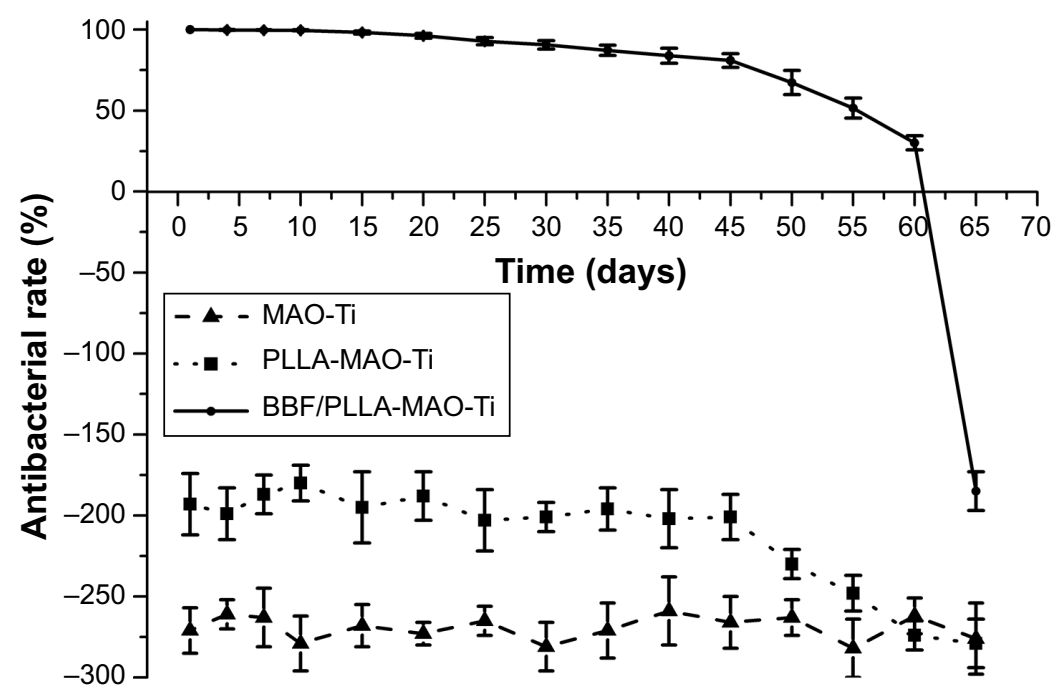

Figure 5 Antibacterial rate for adherent Staphylococcus aureus on the specimens, over 65 days.

Abbreviations: BBF/PLLA-MAO-Ti, cross-linking (Z-)-4-bromo-5-(bromomethylene)-2 $(5 \mathrm{H})$-furanone loaded poly(L-lactic acid) nanoparticles with gelatin on microarcoxidized titanium; MAO-Ti, microarc-oxidized titanium; PLLA-MAO-Ti, cross-linking poly(L-lactic acid) nanoparticles with gelatin on microarc-oxidized titanium. 

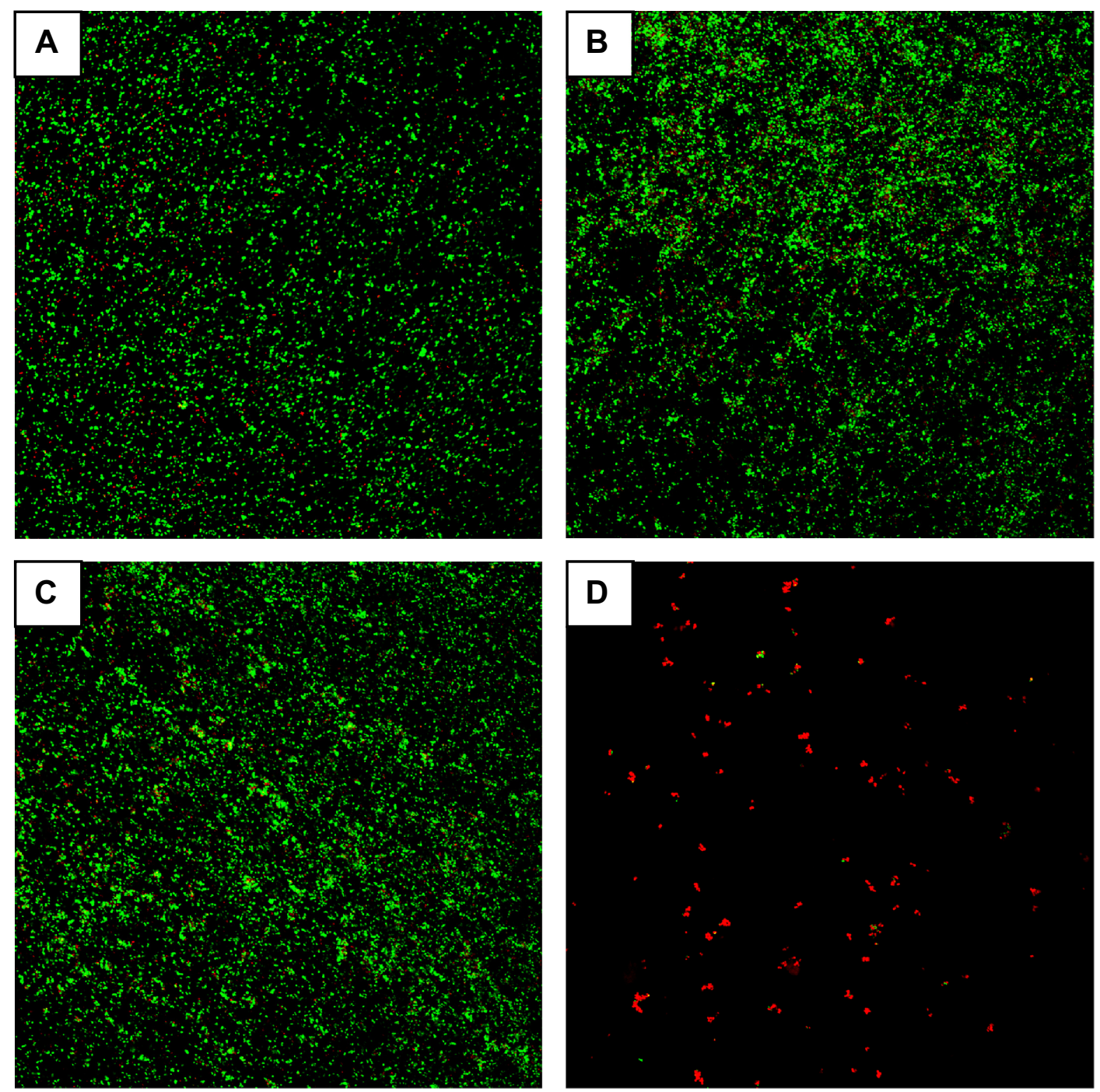

Figure 6 Fluorescence microscopy images showing viability of the adherent Staphylococcus aureus on the specimens, as displayed by SYTO 9 and propidium iodide dyes. (A) polished cp-Ti. (B) MAO-Ti. (C) PLLA-MAO-Ti. (D) BBF/PLLA-MAO-Ti.

Abbreviations: BBF/PLLA-MAO-Ti, cross-linking (Z-)-4-bromo-5-(bromomethylene)-2(5H)-furanone loaded poly(L-lactic acid) nanoparticles with gelatin on microarcoxidized titanium; cp-Ti, commercially pure Ti; MAO-Ti, microarc-oxidized titanium; PLLA-MAO-Ti, cross-linking poly(L-lactic acid) nanoparticles with gelatin on microarcoxidized titanium.

coating (Figure 1F). At the first day, the inhibition zone was biggest, due to the initial burst release of small amounts of BBF. From day 2 to day 45, the released BBF maintained at a steady amount with just a small reduction so there were no obvious changes occurring in the inhibition zone. Subsequently, the inhibition zone shrank markedly, with the significant decrease of released BBF, from day 45 to day 60 . These antibacterial properties are very meaningful for clinical applications. In the early phase after implantation, the local defense system of the host is severely disturbed by the surgical trauma, which makes this the most dangerous time for infection. ${ }^{7}$ The initial burst release, along with the following sustained release of the BBF at a steady amount, helps to prevent postoperative infection, thus ensuring the normal wound healing during the early stage. After this period, because the surgery site has achieved primary healing and osseointegration will be completed gradually over time, a low level of released BBF, conjugated with the host defense, is sufficient to prevent later-stage infection. ${ }^{30}$ In this context, the release profile of BBF and antibacterial characteristics of the novel antibacterial coating fit the proper criteria for clinical application.

The AR value of the antibacterial coating for adherent S. aureus was about $100 \%$ during the first 10 days (Figure 5), which is indicative of the excellent antibacterial ability. Although the AR decreased gradually after 10 days, the AR remained over $90 \%$ in the next 20 days. Of note, the specimens were subject to intense bacterial attack from 
A

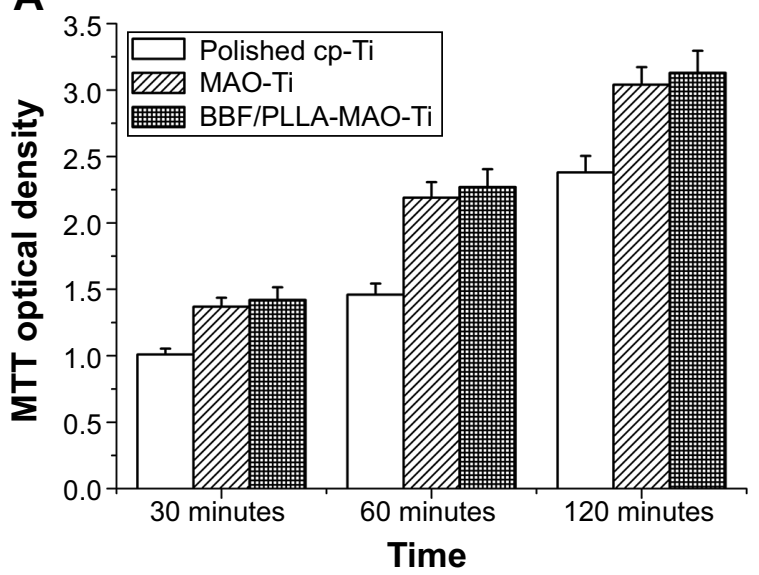

B

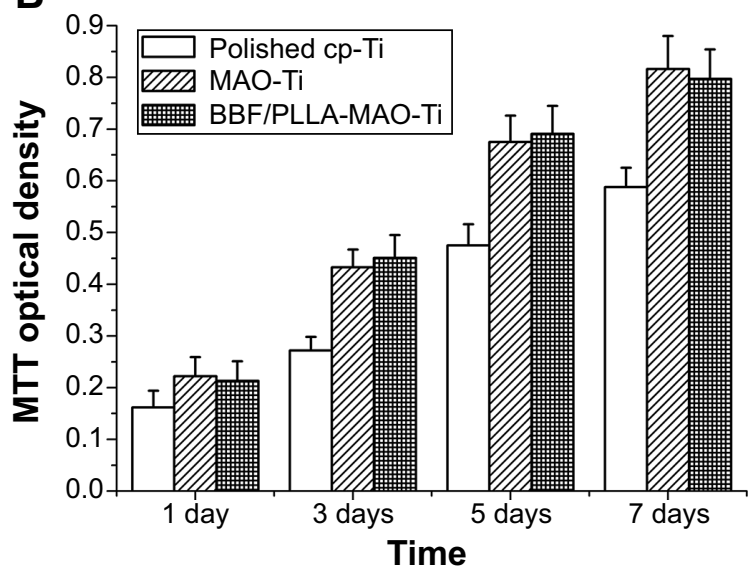

Figure 7 (A) MTT optical density, measured after culture for 30, 60, and I20 minutes. (B) MTT optical density, measured after culture for I, 3 , 5, and 7 days. Abbreviations: BBF/PLLA-MAO-Ti, cross-linking (Z-)-4-bromo-5-(bromomethylene)-2(5H)-furanone loaded poly(L-lactic acid) nanoparticles with gelatin on microarcoxidized titanium; cP-Ti, commercially pure Ti; MAO-Ti, microarc-oxidized titanium; MTT, 3-(4,5-Dimethylthiazol-2-yl)-2,5-diphenyltetrazolium bromide.
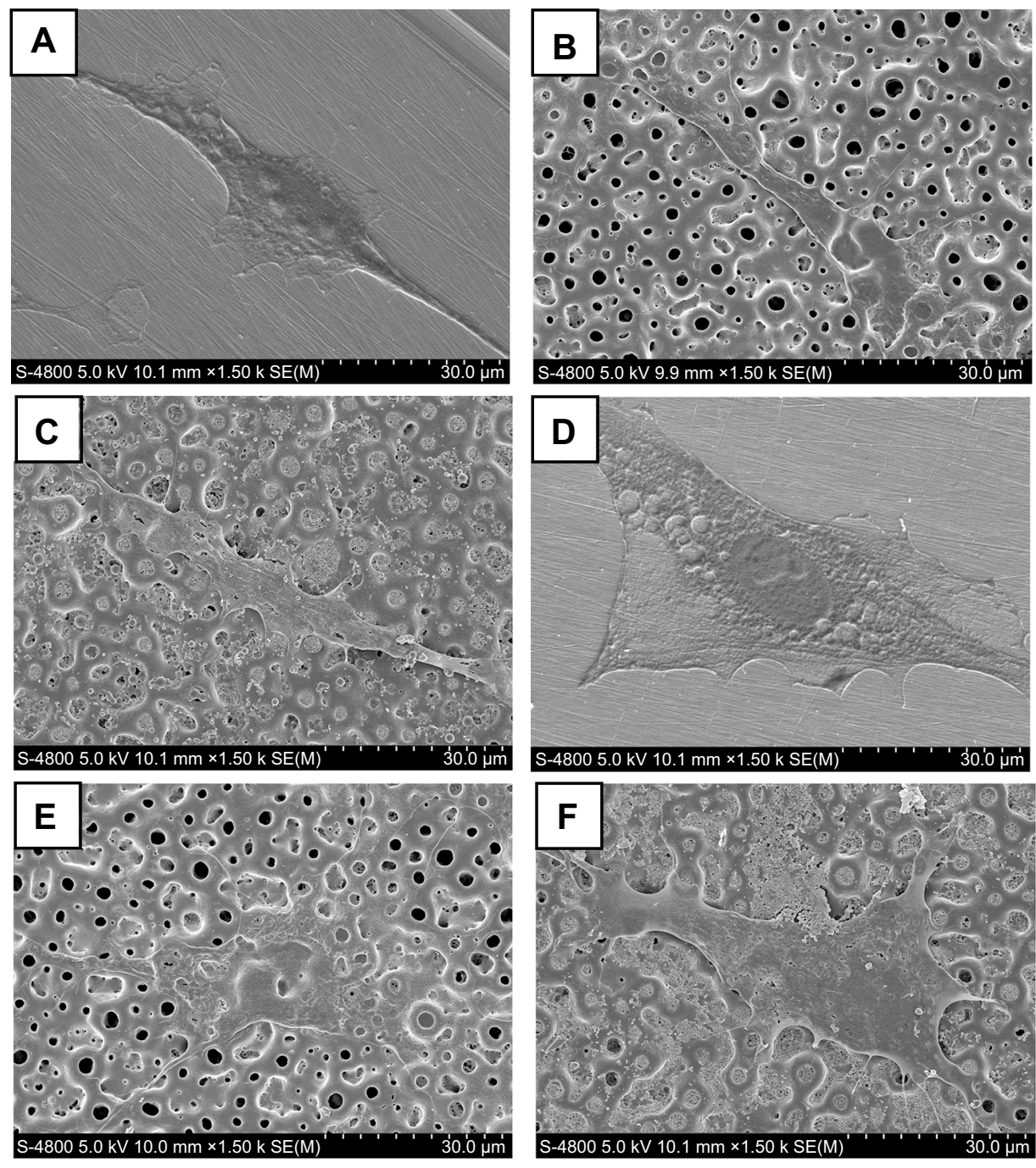

Figure 8 Scanning electron microscope images showing the morphology of osteoblasts spread on the specimens. (A) polished cp-Ti, after I day. (B) MAO-Ti, after I day. (C) BBF/PLLA-MAO-Ti, after I day. (D) polished cp-Ti, after 3 days. (E) MAO-Ti, after 3 days. (F) BBF/PLLA-MAO-Ti, after 3 days.

Abbreviations: BBF/PLLA-MAO-Ti, cross-linking (Z-)-4-bromo-5-(bromomethylene)-2 $(5 \mathrm{H})$-furanone loaded poly(L-lactic acid) nanoparticles with gelatin on microarcoxidized titanium; cP-Ti, commercially pure Ti; MAO-Ti, microarc-oxidized titanium. 
immersion in $1 \mathrm{~mL}$ of the bacteria suspension containing $10^{6} \mathrm{CFU} / \mathrm{mL}$ and changing of the bacteria suspension daily. These conditions were much harsher than the normal situation in vivo. Therefore, the AR value of the antibacterial coating is expected to be higher under normal conditions. The AR values of MAO-Ti and PLLA-MAO-Ti were in the negative range, which indicated that the adherent bacteria on their surfaces were much more than that on polished cp-Ti. Our results were consistent with the previous findings reported by Wang et al. ${ }^{31}$ Studies have proved that roughened surface can encourage bacterial adhesion. ${ }^{32}$ In our study, the roughness of MAO-Ti and PLLA-MAO-Ti was higher than that of polished cp-Ti (Figure 2A). The viability of bacteria on the specimens also confirmed the potent antibacterial ability of the antibacterial coating. Almost no viable bacteria were seen on the antibacterial coating, while large amounts of viable bacteria were observed on the other three specimens (Figure 6).

The results of cell culture experiments showed that MAO-Ti and the antibacterial coating possessed an enhanced biological performance compared with polished cp-Ti. It is known that the morphology and chemical composition of the implant surface determine its interaction with bone. ${ }^{33}$ The improved biological performance of MAO coating has been confirmed repeatedly in the literature. ${ }^{17,24}$ After MAO treatment, a porous surface significantly improved its roughness (Figure 2A). Compared with smooth surface, roughened surface was found to provide osteoblasts with a morphological fixation and thereafter promoted bony ingrowth. ${ }^{24}$ Further, $\mathrm{Ca}$ and $\mathrm{P}$ ions in electrolytes entered the MAO coating (Figure 1C), thus increasing the bioactive potential. ${ }^{34}$ Hydrophilic surface would improve the cell adhesion and spread to the substrates. ${ }^{35}$ The water contact angle on the MAO-Ti was significantly decreased, which suggested that the surface hydrophilicity was substantially improved.

However, it was amazing to observe that the antibacterial coating and the MAO-Ti, showed almost the same biological performance. This may be attributed to two main reasons. First, the application of gelatin in the study may have positively affected the biological performance. It is known that gelatin is created through either acid or alkaline hydrolysis of collagen and has conventionally been employed for pharmaceutical, food, and cosmetic products. ${ }^{36}$ The hydrolysis process hydrolyzes the amide structures of asparagine or glutamine side chains, generating a high percentage of carboxylic groups and amino groups, ${ }^{37}$ which increases the hydrophilicity. So in this study, the antibacterial coating showed a higher hydrophilicity (Figure 2B). On the other hand, although the BBF-PLLA-NPs were attached in the pores of the MAO coating, the $\mathrm{Ca}$ - and $\mathrm{P}$-enriched junction areas among the pores still increased the bioactivity. The $\mathrm{Ca} / \mathrm{P}$ ratio of the MAO coating was 1.54 , very close to that of the hydroxylapatite (1.67), so the bone-like apatite that formed made the surface more bioactive.

It has been reported that the ideal design of antibacterial coatings on $\mathrm{Ti}$ implants should meet certain requirements: (1) the selected antibacterial agent should not promote the development of drug resistance; (2) the release should be sustained; and (3) the biocoatings should be osteoconductive. ${ }^{38}$ In our study, BBF was a member of the halogenated furanone compounds, with strong antibacterial ability and low risk of resistance development. This antibacterial coating was able to achieve sustained release of BBF for 60 days and possessed relatively long-term antibacterial effects. Further, the antibacterial coating showed an improved biological activity compared with polished cp-Ti. Taken together, the fabricated antibacterial coating may serve as an ideal approach to prevent implant-associated infection.

\section{Conclusion}

A novel antibacterial coating containing BBF-PLLA-NPs was fabricated on MAO-Ti. The antibacterial coating produced a unique inhibition zone against $S$. aureus throughout the 60-day period, and the AR for adherent bacteria was about $100 \%$ in the first 10 days. Results of fluorescence staining of adherent $S$. aureus also confirmed the excellent antibacterial ability of the antibacterial coating. Moreover, the antibacterial coating showed an enhanced osteoblast adhesion, proliferation, and more notable cell spread during the early stage. It can be concluded that use of the fabricated antibacterial coating, with its relatively long-term antibacterial ability and excellent biological performance, may be a potential and promising strategy to prevent implantassociated infection.

\section{Acknowledgment}

This work was supported by the National Natural Science Foundation of China (grant numbers 51001116 and 51371006).

\section{Disclosure}

The authors report no conflicts of interest in this work.

\section{References}

1. Niinomi M. Mechanical biocompatibilities of titanium alloys for biomedical applications. J Mech Behav Biomed Mater. 2008;1(1):30-42.

2. Darouiche RO. Treatment of infections associated with surgical implants. N Engl J Med. 2004;350(14):1422-1429. 
3. Zimmerli W, Trampuz A, Ochsner PE. Prosthetic-joint infections. N Engl J Med. 2004;351(16):1645-1654.

4. Hetrick EM, Schoenfisch MH. Reducing implant-related infections: active release strategies. Chem Soc Rev. 2006;35(9):780-789.

5. Hu H, Zhang W, Qiao Y, Jiang X, Liu X, Ding C. Antibacterial activity and increased bone marrow stem cell functions of $\mathrm{Zn}$-incorporated $\mathrm{TiO}_{2}$ coatings on titanium. Acta Biomater. 2012;8(2):904-915.

6. Necula BS, Fratila-Apachitei LE, Zaat SA, Apachitei I, Duszczyk J. In vitro antibacterial activity of porous $\mathrm{TiO}_{2}-\mathrm{Ag}$ composite layers against methicillin-resistant Staphylococcus aureus. Acta Biomater. 2009;5(9):3573-3580.

7. Zhao L, Chu PK, Zhang Y, Wu Z. Antibacterial coatings on titanium implants. J Biomed Mater Res B Appl Biomater. 2009;91(1):470-480.

8. Tunney MM, Ramage G, Patrick S, Nixon JR, Murphy PG, Gorman SP. Antimicrobial susceptibility of bacteria isolated from orthopedic implants following revision hip surgery. Antimicrob Agents Chemother. 1998;42(11):3002-3005.

9. Baveja JK, Willcox MD, Hume EB, Kumar N, Odell R, Poole-Warren LA. Furanones as potential anti-bacterial coatings on biomaterials. Biomaterials. 2004;25(20):5003-5012.

10. Manefield M, Rasmussen TB, Henzter M, et al. Halogenated furanones inhibit quorum sensing through accelerated LuxR turnover. Microbiology. 2002;148(Pt 4):1119-1127.

11. Raffa RB, Iannuzzo JR, Levine DR, et al. Bacterial communication ("quorum sensing") via ligands and receptors: a novel pharmacologic target for the design of antibiotic drugs. $J$ Pharmacol Exp Ther. 2005;312(2):417-423.

12. Lönn-Stensrud J, Landin MA, Benneche T, Petersen FC, Scheie AA. Furanones, potential agents for preventing Staphylococcus epidermidis biofilm infections? J Antimicrob Chemother. 2009;63(2):309-316.

13. Baveja JK, Li G, Nordon RE, et al. Biological performance of a novel synthetic furanone-based antimicrobial. Biomaterials. 2004; 25(20):5013-5021

14. Dalpiaz A, Scatturin A, Pavan B, Biondi C, Vandelli MA, Forni F. Poly(lactic acid) microspheres for the sustained release of a selective A1 receptor agonist. J Control Release. 2001;73(2-3):303-313.

15. Kashi TS, Eskandarion S, Esfandyari-Manesh M, et al. Improved drug loading and antibacterial activity of minocycline-loaded PLGA nanoparticles prepared by solid/oil/water ion pairing method. Int $J$ Nanomedicine. 2012;7:221-234.

16. Madhavan Nampoothiri K, Nair NR, John RP. An overview of the recent developments in polylactide (PLA) research. Bioresour Technol. 2010;101(22):8493-8501.

17. Wu J, Liu ZM, Zhao XH, Gao Y, Hu J, Gao B. Improved biological performance of microarc-oxidized low-modulus Ti-24Nb-4Zr-7.9Sn alloy. J Biomed Mater Res B Appl Biomater. 2010;92(2):298-306.

18. Yao ZQ, Ivanisenko Y, Diemant T, et al. Synthesis and properties of hydroxyapatite-containing porous titania coating on ultrafine-grained titanium by micro-arc oxidation. Acta Biomater. 2010;6(7):2816-2825.

19. Song WH, Jun YK, Han Y, Hong SH. Biomimetic apatite coatings on micro-arc oxidized titania. Biomaterials. 2004;25(17):3341-3349.

20. Wei D, Zhou Y, Jia D, Wang Y. Characteristic and in vitro bioactivity of a microarc-oxidized $\mathrm{TiO}(2)$-based coating after chemical treatment. Acta Biomater. 2007;3(5):817-827.

21. Yao J, Cheng Y, Zhao W, Zheng Z, Gao B, Wu J. Preparation and in vitro release behavior of halogenated furanone polylactic acid microsphere. J Pract Stomatol. 2013;4:71-75.

22. Cheng Y, Wu J, Gao B, et al. Fabrication and in vitro release behavior of a novel antibacterial coating containing halogenated furanone-loaded poly(L-lactic acid) nanoparticles on microarc-oxidized titanium. Int $J$ Nanomedicine. 2012;7:5641-5652.

23. Shameli K, Ahmad MB, Zargar M, Yunus WM, Rustaiyan A, Ibrahim NA. Synthesis of silver nanoparticles in montmorillonite and their antibacterial behavior. Int J Nanomedicine. 2011;6:581-590.

24. Zhao L, Wei Y, Li J, Han Y, Ye R, Zhang Y. Initial osteoblast functions on Ti-5Zr-3Sn-5Mo-15Nb titanium alloy surfaces modified by microarc oxidation. J Biomed Mater Res A. 2010;92(2):432-440.

25. Fielding GA, Roy M, Bandyopadhyay A, Bose S. Antibacterial and biological characteristics of silver containing and strontium doped plasma sprayed hydroxyapatite coatings. Acta Biomater. 2012;8(8): 3144-3152.

26. Stigter M, Bezemer J, de Groot K, Layrolle P. Incorporation of different antibiotics into carbonated hydroxyapatite coatings on titanium implants, release and antibiotic efficacy. J Control Release. 2004; 99(1):127-137.

27. Janssens JC, Steenackers H, Robijns S, et al. Brominated furanones inhibit biofilm formation by Salmonella enterica serovar Typhimurium. Appl Environ Microbiol. 2008;74(21):6639-6648.

28. Lee JW, Gardella JA, Hicks W, Hard R, Bright FV. Analysis of the initial burst of drug release coupled with polymer surface degradation. Pharm Res. 2003;20(2):149-152.

29. Lee WK, Park JY, Yang EH, et al. Investigation of the factors influencing the release rates of cyclosporin A-loaded micro- and nanoparticles prepared by high-pressure homogenizer. J Control Release. 2002;84(3):115-123.

30. Zhao L, Wang H, Huo K, et al. Antibacterial nano-structured titania coating incorporated with silver nanoparticles. Biomaterials. 2011;32(24):5706-5716

31. Wang X, Wang G, Liang J, Cheng J, Ma W, Zhao Y. Staphylococcus aureus adhesion to different implant surface coatings: An in vitro study. Surf Coat Tech. 2009;203(22):3454-3458.

32. Harris LG, Richards RG. Staphylococcus aureus adhesion to different treated titanium surfaces. J Mater Sci Mater Med. 2004;15(4): 311-314.

33. Rungsiyakull C, Li Q, Sun G, Li W, Swain MV. Surface morphology optimization for osseointegration of coated implants. Biomaterials. 2010;31(27):7196-7204.

34. Schreckenbach JP, Marx G, Schlottig F, Textor M, Spencer ND. Characterization of anodic spark-converted titanium surfaces for biomedical applications. J Mater Sci Mater Med. 1999;10(8):453-457.

35. Bhattarai SR, Bhattarai N, Viswanathamurthi P, Yi HK, Hwang PH, Kim HY. Hydrophilic nanofibrous structure of polylactide; fabrication and cell affinity. J Biomed Mater Res A. 2006;78(2):247-257.

36. Frutos G, Prior-Cabanillas A, París R, Quijada-Garrido I. A novel controlled drug delivery system based on $\mathrm{pH}$-responsive hydrogels included in soft gelatin capsules. Acta Biomater. 2010;6(12):4650-4656.

37. Lee KY, Mooney DJ. Hydrogels for tissue engineering. Chem Rev. 2001; 101(7):1869-1879.

38. Kazemzadeh-Narbat M, Lai BF, Ding C, Kizhakkedathu JN, Hancock RE, Wang R. Multilayered coating on titanium for controlled release of antimicrobial peptides for the prevention of implant-associated infections. Biomaterials. 2013;34(24):5969-5977.

\section{Dovepress}

\section{Publish your work in this journal}

The International Journal of Nanomedicine is an international, peerreviewed journal focusing on the application of nanotechnology in diagnostics, therapeutics, and drug delivery systems throughout the biomedical field. This journal is indexed on PubMed Central, MedLine, CAS, SciSearch ${ }^{\circledR}$, Current Contents ${ }^{\circledR} /$ Clinical Medicine,

Journal Citation Reports/Science Edition, EMBase, Scopus and the Elsevier Bibliographic databases. The manuscript management system is completely online and includes a very quick and fair peer-review system, which is all easy to use. Visit http://www.dovepress.com/ testimonials.php to read real quotes from published authors. 